\title{
All-fiber all-normal dispersion laser with a fiber-based Lyot filter
}

\author{
K. Özgören ${ }^{1, *}$ and F. Ö. Ilday ${ }^{2}$ \\ ${ }^{1}$ Material Science and Nanotechnology Graduate Program, Bilkent University, Ankara 06800, Turkey \\ ${ }^{2}$ Physics Department, Bilkent University, Çankaya, Ankara 06800, Turkey \\ *Corresponding author: kozgoren@fen.bilkent.edu.tr
}

Received January 11, 2010; revised March 12, 2010; accepted March 16, 2010; posted March 19, 2010 (Doc. ID 122609); published April 15, 2010

\begin{abstract}
We propose the use of a short section of polarization-maintaining fiber as a birefringent medium to construct an all-fiber Lyot filter inside the cavity of a fiber laser. This allows mode-locked operation of an all-fiber all-normal dispersion Yb-fiber oscillator without the use of a bulk bandpass filter and using standard components. Moreover, filter bandwidth and modulation depth is easily controlled by changing the length and splice angle of the polarization-maintaining-fiber section, leading to an adjustable filter. At mode-locked operation, the $30 \%$ output fiber port delivers $1 \mathrm{~nJ}$ pulses that are dechirped to $230 \mathrm{fs}$ duration. () $2010 \mathrm{Op}$ tical Society of America

OCIS codes: $140.3510,140.4050,140.7090$.
\end{abstract}

In recent years, all-normal-dispersion fiber lasers working in the ytterbium wavelength region have attracted much interest because of their simpler cavities compared with other types of fiber lasers [1,2]. The large chirp on the pulse minimizes the nonlinear effects and allows high-energy operation. The modelocked operation is described well by dissipative soliton formation [3]. In practice, the use of an interference or birefringent filter instead of a grating pair amounts to a certain simplification of the cavity. However, these filters are bulk components, whereas an all-fiber oscillator design is attractive, as it would be misalignment free. Various approaches have been pursued to build an all-fiber cavity near $1 \mu \mathrm{m}$. Prochnow et al. [4] used a saturable Bragg reflector along with nonlinear polarization evolution (NPE) for building an all-fiber cavity and obtained $0.8 \mathrm{~nJ}, 10 \mathrm{ps}$ pulses compressible up to $627 \mathrm{fs}$. Nielsen et al. [5] used a fiber Bragg grating for spectral filtering in their laser, which is mainly based on nonlinear polarization rotation. Their pulse energy was $1.3 \mathrm{~nJ}$ with 5.6 ps duration. Kieu et al. [6] used a wavelength division multiplexing (WDM) based filter along with a carbon nanotube saturable absorber and obtained $3 \mathrm{~nJ}, 1.5 \mathrm{ps}$ pulses that could be dechirped to $250 \mathrm{fs}$. Schultz et al. [7] also used a WDM based filter along with NPE producing $1.8 \mathrm{~nJ}, 7.6 \mathrm{ps}$ pulses that can be dechirped to $179 \mathrm{fs}$. Lastly, Fekete et al. [8] used a semiconductor saturable absorber along with NPE in order to obtain $0.2 \mathrm{~nJ}, 10 \mathrm{ps}$ pulses compressible to $200 \mathrm{fs}$.

Here, we demonstrate theoretically and experimentally that a short section of polarizationmaintaining (PM) fiber placed after a polarizer mimics a Lyot filter [9], allowing the construction of an all-fiber all-normal-dispersion $\mathrm{Yb}$-doped fiber laser using standard components. NPE [10] is utilized as an effective saturable absorber to start and stabilize the mode-locked operation.

A Lyot filter is ordinarily composed of two polarizers with a birefringent plate at an angle of $45^{\circ}$ sandwiched in between. The first polarizer imposes linear polarization, which is decomposed into orthogonal states as it couples to the fast and slow axes of the birefringent medium. The birefringence leads to the accumulation of a relative phase difference, $\Delta \varphi$, between the beams propagating along the slow and fast axes, which corresponds to wavelength-dependent rotation of the polarization state. The second polarizer converts this rotation into amplitude modulation, and the transmittance is given by $T=\cos ^{2}(\Delta \varphi / 2)$. Thus a spectral filter with cosine-square profile is obtained [11].

In our all-fiber-integrated scheme, PM fiber output of an in-fiber polarizing beam splitter (PBS) is spliced to a length of PM fiber, which acts as the birefringent plate. The axes of the two PM fibers are spliced at an angle (nominally $45^{\circ}$ ), thereby coupling light into both axes of the PM fiber. Ordinarily this should be followed by a second PBS; however, it is not needed for a ring cavity configuration, since the first PBS delivers the function of the second polarizer at the end of one round trip. A schematic of the laser cavity is shown in Fig. 1, with the polarization evolution indicated. The bandwidth, central wavelength, and the modulation depth of the filter are in principle controllable. The phase difference accumulated between the fast and slow axes over a section of PM fiber is given

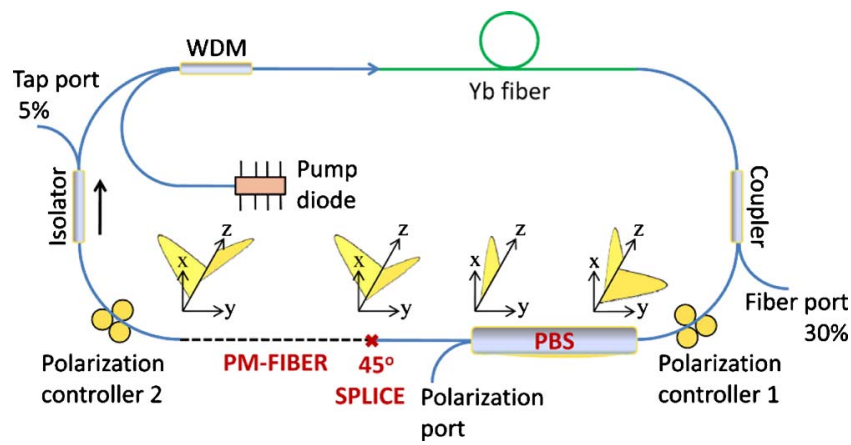

Fig. 1. (Color online) Sketch of the setup. The Lyot filter action is illustrated. Fast axis of the PM fiber makes a $45^{\circ}$ angle with the polarizer. The $z$ axis is along the fiber direction. 
by $\Delta \varphi=(2 \pi / \lambda) L \Delta n$, where $L$ is the length of the PM fiber, $\lambda$ is the wavelength, and $\Delta n=n_{\text {slow }}-n_{\text {fast }}$ is the birefringence. The boundary conditions imposed by the cavity will be the same if the phase difference $\Delta \varphi$ accumulated in the PM-fiber section is a multiple of $2 \pi$. Two successive wavelengths satisfying this condition are $\lambda_{m}=L \Delta n / m$ and $\lambda_{m+1}=L \Delta n /(m+1)$, where $m$ is an integer. Assuming $m \gg 1$, the peak-to-peak filtering bandwidth, $\Delta \lambda$, of the PM-fiber section is given by

$$
\Delta \lambda=\lambda_{m}-\lambda_{m+1}=L \Delta n\left(\frac{1}{m}-\frac{1}{m+1}\right) \cong \frac{\lambda^{2}}{L \Delta n} .
$$

Thus the bandwidth can be controlled through the length of the PM fiber section. After the PM fiber, the beam undergoes polarization rotations through the rest of the cavity until it reaches to the PBS again. A simplified analysis of the cavity based on Jones matrices, beginning at the PBS, can be constructed to illustrate the key properties of the filter:

$$
\left[\begin{array}{cc}
e^{i \beta} \cos \alpha & \sin \alpha \\
-e^{i \beta} \sin \alpha & \cos \alpha
\end{array}\right] \times\left[\begin{array}{cc}
e^{i \Delta \varphi} \cos ^{2} \theta+\sin ^{2} \theta & 0 \\
\left(e^{i \Delta \varphi}-1\right) \sin \theta \cos \theta & 0
\end{array}\right],
$$

where the right matrix represents the combined effect of the PBS and the PM fiber, which is spliced at an angle of $\theta$. The modulation depth of the filter is determined by the ratio of light coupled into the slow and fast axes of the PM fiber and is maximized for $45^{\circ}$ angle. The left matrix represents the polarization rotations in the rest of the cavity, which is taken as an arbitrary rotation $(\alpha)$ and retardation $(\beta)$. For maximum modulation depth, $\theta=45^{\circ}$, and the resultant matrix simplifies to

$$
\frac{1}{2}\left[\begin{array}{cc}
\left(e^{i \Delta \varphi}+1\right) e^{i \beta} \cos \alpha+\left(e^{i \Delta \varphi}-1\right) \sin \alpha & 0 \\
-\left(e^{i \Delta \varphi}+1\right) e^{i \beta} \sin \alpha+\left(e^{i \Delta \varphi}-1\right) \cos \alpha & 0
\end{array}\right] .
$$

The peak transmission wavelength is given by the condition for maximizing the horizontal component. Differentiating it with respect to $\Delta \varphi$ and equating to zero yields

$$
\tan \Delta \varphi=-\tan (2 \alpha) \sin \beta
$$

For $\alpha, \beta=0$, it reduces to an ordinary Lyot filter, and the maximum transmission occurs at $\Delta \varphi=2 \pi m$, where $m=0,1,2, \ldots$. For different $\alpha$ and $\beta$ values, peak transmission wavelength shifts, but the filter bandwidth is unchanged. Thus the peak transmission wavelength can be controlled by polarization controllers, though this degree of freedom is reserved for NPE operation and offers little practical value.

Although the operation of a PM fiber implementation of the Lyot filter is straightforward for a cw laser, its applicability to mode-locked operation is not clear a priori. In addition to the phase-difference accumulation, there is temporal walk-off due to the difference in group velocities along the PM fiber, which can destabilize mode-locked operation by breaking a pulse into two components. We have resorted to numerical simulations based on the model described in
[12] to address these questions using parameters that correspond to the experiments described below. The effect of the walk-off is modeled by splitting the field into two components corresponding to the fast and slow axes of the PM fiber. The slow component is temporally delayed, then the components are added together, similar to the analysis in [13]. We find that maximum tolerable walk-off increases with decreasing modulation depth of the filter. For maximum modulation depth, stable operation is attained for up to $\sim 600$ fs temporal walk-off, which corresponds to a minimum (peak-to-peak) filter bandwidth of approximately $6 \mathrm{~nm}$. Figure 2 shows the simulation results obtained with the PM fiber filter, in comparison with ordinary filters with cosine-square and Gaussian shapes.

Encouraged by the simulation results, we constructed an all-normal-dispersion Yb-fiber laser oscillator (Fig. 1). A $980 \mathrm{~nm}$ diode pumps a highly doped 30-cm-long Yb-fiber through a WDM. Approximately $30 \%$ of the beam is extracted from the cavity through a fiber coupler placed after the gain fiber. The rest of the light is sent to the PBS. A section of PM fiber is spliced at $45^{\circ}$ angle to the PM lead fiber of the PBS. All the other fibers in the cavity are non-PM. An inline isolator ensures unidirectional operation and has a 5\% output port for monitoring purposes. Two in-line polarization controllers are used to control the polarization. Since a number of cavity elements can effectively function as unintended filters, it is important to verify that the PM-fiber filter is the dominant filtering mechanism. This is confirmed by $\mathrm{cw}$ operation of the laser, which operates only at distinct wavelengths, with fixed distances between the peaks (Fig. $3)$. The peaks shift all together when the polarization state is changed via polarization controllers, as predicted by Eq. (4). For two different PM fiber lengths, namely $37 \mathrm{~cm}$ and $24 \mathrm{~cm}$, the measured (peak-topeak) bandwidths are $6.5 \mathrm{~nm}$ and $10.0 \mathrm{~nm}$, respec-
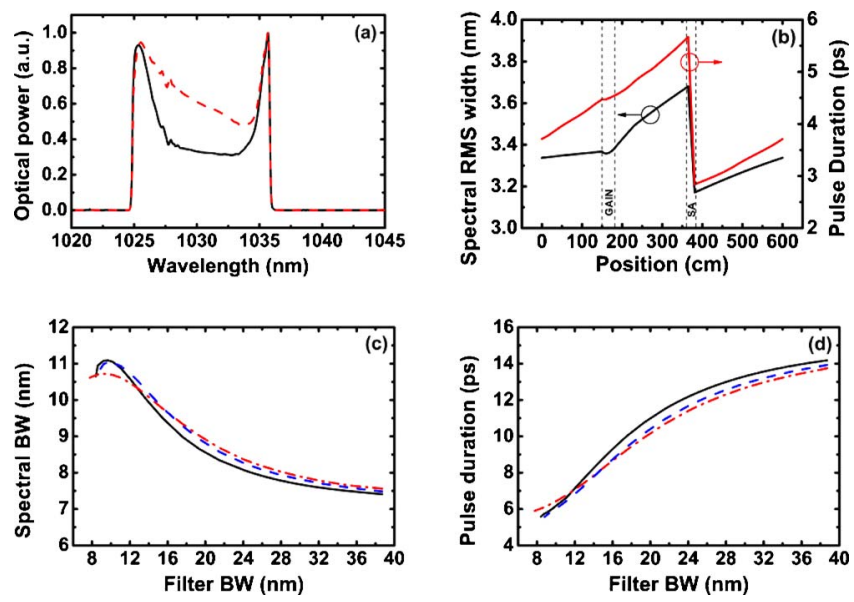

Fig. 2. (Color online) (a) Simulated laser spectrum after the gain fiber (solid black curve) and after the PM fiber (red dashed curve). (b) Simulated spectral bandwidth (solid black curve) and pulse duration [solid gray (red) curve] variation over the cavity. Comparison of (c) the spectral width and (d) the pulse duration obtained numerically with the PM-fiber filter (solid black curves), Gaussian filter (red dashed-dotted curve) and cosine-square filter (blue dashed curves) 

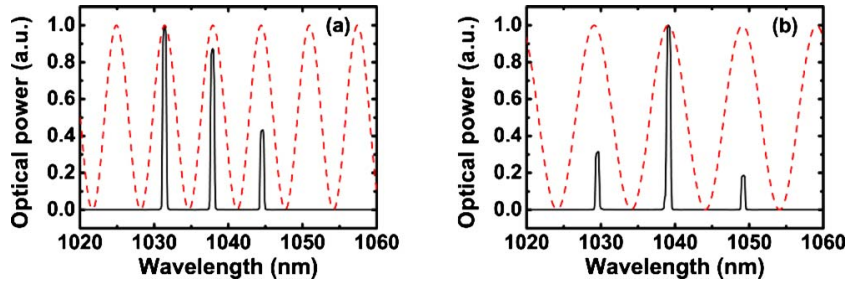

Fig. 3. (Color online) Measured optical spectra for cw operation (solid black curve) and theoretical transmission curves (red dashed curves) for effective filter bandwidths of (a) $6.5 \mathrm{~nm}$ and (b) $10.0 \mathrm{~nm}$.

tively. These values precisely match the inverse scaling of bandwidth with fiber length. The calculated birefringence of the fiber is $\Delta n=4.4 \times 10^{-4}$, which is close to the beat length specified as $\leq 2.7 \mathrm{~mm}$ by the manufacturer.

For the remainder of the work, we set the PM-fiber length to $28 \mathrm{~cm}$, which corresponds to $8.6 \mathrm{~nm}$ filter bandwidth and $410 \mathrm{fs}$ of temporal walk-off. The laser is mode-locked easily by adjusting the polarization controllers. The output power of the laser is $33 \mathrm{~mW}$ at the $30 \%$ fiber port and $50 \mathrm{~mW}$ at the polarization port at a repetition rate of $34 \mathrm{MHz}$, which corresponds to $1.0 \mathrm{~nJ}$ and $1.5 \mathrm{~nJ}$ of pulse energy at the fiber and polarization ports, respectively. The intracavity pulse energy is $3.3 \mathrm{~nJ}$ after the gain fiber. Figure 4 shows the spectra of the laser obtained at different ports, showing that the spectral filtering is taking place primarily at the PBS, as expected. The laser produces chirped pulses, which are compressed externally using a grating compressor, giving a minimum pulse duration of $230 \mathrm{fs}$, assuming a Gaussian decon-
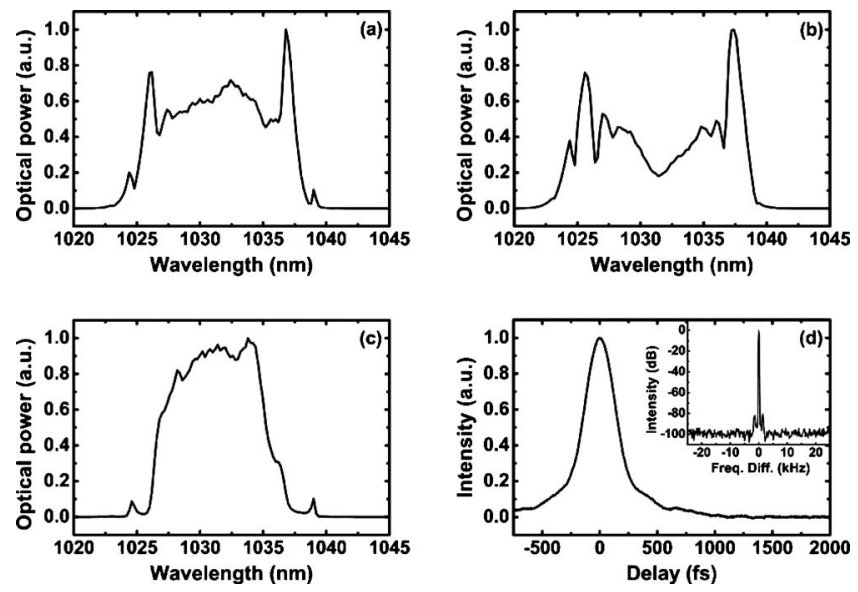

Fig. 4. Measured spectra of pulses for $8.6 \mathrm{~nm}$ effective filter bandwidth from (a) the $30 \%$ fiber port, (b) the polarization extraction port, (c) the 5\% port. (d) Measured autocorrelation of the pulses from the $30 \%$ fiber port. Inset, rf spectrum of the pulses. volution factor. The autocorrelation trace of the compressed pulses and high-dynamic-range rf spectrum are shown in Fig. 4(d). The rf spectrum of the laser shows $90 \mathrm{~dB}$ suppression of the sidebands, which are $1.5 \mathrm{kHz}$ apart. There is no sign of multiple-pulsing at this energy level, which is limited by the maximum available pump power of $300 \mathrm{~mW}$.

In conclusion, we demonstrate that a PM-fiber section inserted into an all-normal-dispersion laser cavity functions as a Lyot filter and stable mode-locked operation is possible, which is confirmed experimentally and numerically. The filter bandwidth and modulation depth are adjustable through the length and splice angle of the PM-fiber section, respectively. We have demonstrated an all-fiber $\mathrm{Yb}$-doped laser cavity without using specialty components such as semiconductor saturable absorbers or chirped fiber Bragg gratings for the first time to our knowledge. The laser generates 1.0 and $1.5 \mathrm{~nJ}$-energy pulses from its two ports. The pulses can be dechirped to $\sim 230$ fs. Low-noise operation is confirmed by highdynamic-range rf spectrum measurements.

This work was supported by the Scientific and Technological Research Council of Turkey (TÜBITAK) under grant 106G089, SAN-TEZ under grant 00255.STZ.2008-1, EU Marie Curie IRG FiberLaser, Bilkent University Research Funds, and by the Distinguished Young Scientist Award of the Turkish Academy of Sciences (TÜBA).

\section{References}

1. A. Chong, J. Buckley, W. Renninger, and F. Wise, Opt. Express 14, 10095 (2006).

2. F. W. Wise, A. Chong, and W. H. Renninger, Laser Photonics Rev. 2, 58 (2008).

3. W. H. Renninger, A. Chong, and F. W. Wise, Phys. Rev. A 77, 023814 (2008).

4. O. Prochnow, A. Ruehl, M. Schultz, D. Wandt, and D. Kracht, Opt. Express 15, 6889 (2007).

5. C. K. Nielsen and S. R. Keiding, Opt. Lett. 32, 1474 (2007).

6. K. Kieu and F. W. Wise, Opt. Express 16, 11453 (2008).

7. M. Schultz, H. Karow, O. Prochnow, D. Wandt, U. Morgner, and D. Kracht, Opt. Express 16, 19562 (2008).

8. J. Fekete, A. Cserteg and R. Szipocs, Laser Phys. Lett. 6, 49 (2009).

9. B. Lyot, C. R. Acad. Sci. III 197, pp. 1593 (1933).

10. M. Hofer, M. E. Fermann, F. Harberl, M. H. Ober, and A. J. Schmidt, Opt. Lett. 16, 502 (1991).

11. G. Shabtay, E. Eidinger, Z. Zalevsky, D. Mendlovic and E. Marom, Opt. Express 10, 1534 (2002).

12. P. K. Mukhopadhyay, K. Özgören, I. L. Budunoglu, and F. Ö. Ilday, IEEE J. Sel. Top. Quantum Electron. 15, 145 (2009).

13. H. Lim, F. Ö. Ilday, and F. W. Wise, Opt. Express 10, 1497 (2002). 